\title{
Cardiovascular involvement in Pompe disease
}

\author{
Alina-Costina Luca ${ }^{1}$, Elena Braha² \\ ${ }^{1}$ Pediatric Cardiology Clinic, "Sf. Maria" Pediatric Hospital, "Gr. T. Popa” University of Medicine and \\ Pharmacy, lasi, Romania \\ ${ }^{2}$ Medical Genetics Department, "Sf. Maria" Pediatric Hospital, "Gr. T. Popa" University of Medicine \\ and Pharmacy, Iasi, Romania
}

\begin{abstract}
Lysosomal storage diseases are a diverse group of monogenic disorders which are as defined by defects in lysosomal function. The heart is part of the clinical phenotype of lysosomal storage diseases. Pompe disease is marked by absence/deficiency of the lysosomal enzyme alpha-glucosidase and by different ages of onset. The infantile form is defined by muscle weakness and progressive cardiac hypertrophy, followed by progressive cardiac failure. Pompe disease requires immediate intervention to maximize the potential benefit from enzymatic therapy, with improvement of the phenotype. This article presents clinical and cardiac findings suggestive for Pompe's disease.
\end{abstract}

Keywords: Iysosomal diseases, Pompe disease, cardiomyopathy

\section{Lysosomal storage diseases}

Lysosomes are single phospholipid bilayer membrane cytoplasmatic organelles which contain hydrolytic enzymes. Lysosomal storage diseases (LSD) are a diverse group of monogenic disorders (mostly recessively inherited, few X-linked) which are the consequence of defects in lysosomal function, defined by the accumulation of undigested/ partially digested macromolecules, which produce cellular dysfunction and secondary, a multi-systemic pathology (1).

LSD have a frequency of about 1/8000 live births (2).

LSD are mostly produce by the lack of lysosomal hydrolase but defects in post-translational modification proteins, activator proteins or lysosomal trafficking proteins have been reported recently (3).

Recently LSD have been classified according to the molecular defect detect to the patient (defects in glycan degradation, in lipid degradation, in protein degradation, in lysosomal transporters, in lysosomal trafficking) $(4,5)$.

From the clinical point of view the combination of dysmorphic features (coarse facies, macroglossia), bone anomalies (dysostosis), heart anomalies (arrhythmia, cardiomegaly), hepatosplenomegaly and neurological symptoms (developmental delay, hypotonia, epilepsy, peripheral neuropathy, mental retardation, ataxia/spasticity) may strongly suggest the clinical suspicion of LSD. Classically the symptomes are progressive (6).

The enzymatic test is possible for most LSD. Molecular genetic testing can confirm the enzymatic diagnosis and can clarify the type of genetic variation which is an important information in evaluating enzyme replacement therapy (4).

Genetic counselling for at-risk couples includes prenatal testing on chorionic villi or amniocytes. LSD are inherited as autosomal recessive diseases, except for Fabry disease, Hunter syndrome and Danon disease which are X-linked disorders (4).

\section{Pompe disease}

Pompe disease (glycogen storage disease II) is an autosomal recessive disease, with multisystemic and progressive neuromuscular hypotonie, caused by a mutation in the acid alpha-1,4-glucosidase gene (chromosome 17q25.3) (7).

The estimated incidence of Pompe disease (PD) is $1 / 40,000$ (8). The adult form of PD has an incidence of 1/57,000 newborn per year (9). 
The incidence of carrier is $1 / 138,000$ for classic infantile disease and 1/57,000 for late-onset disease (10).

The infantile onset PD appears to be more common in African-Americans, in China and Taiwan (11) while the adult form is more frequent in the Netherlands (8).

In classic form of PD with infantile onset the patient has severe hypotonie (20-63\% cases), cardiac anomalies with congestive heart failure (50$92 \%$ cases), macroglossia, feeding difficulties (44$97 \%$ cases $)$ and respiratory problems $(27-78 \%$ cases) (12-14).

The non-classic variant of infantile-onset PD is manifested in the first year of life as motor delays and progressive muscular hypotonie. Usually the cardiac involvement is mild. $(14,15)$.

Late-onset of PD could be considered in patients with proximal muscular weakness and respiratory insufficiency, with no apparent cardiac involvement (15).

This form manifests after 27-41 years but the early disease manifestations prevail the diagnosis with 3-10 years because the slow and relatively unspecific progression of symptoms $(16,17)$.

Clinical findings should always be confirmed by additional tests. Nonspecific tests include serum creatine kinase (which is elevated in classic form of PD and normal in adult onset PD) (18) and urinary glucose tetrasaccharide which is highly sensitive for PD but nonspecific (19).

The specific tests are acid alpha-glucosidase (GAA) enzyme activity in blood dried samples which has a low activity. The test is performed before to initiation of therapy. GAA enzyme activity in cultured skin fibroblasts or muscle is the standard test but difficult to perform. $(20 ; 18)$ The molecular test may help confirm a PD diagnosis and this test is especially used in families where there was already a PD case and is trying to identify carriers of the mutated gene in order to provide genetic counseling (18).

Enzyme replacement therapy with recombinant human acid $\alpha$-glucosidase showed an extension of survival and an improvement in cardiac function (21). Some studies revealed that $\beta$-Blockers, such as propranolol, may reduce the efficiency of enzymatic treatment by modulating the receptor expression (22).

From the point of view of cardiologists, PD is an important cause of familial hypertrophic cardiomyopathy (23). Cardiac structure and function should be evaluated through echocardiography, EKG and Holter monitoring (24).
The classic infantile form of PD is characterised by the presence of hypertrophic cardiomyopathy (septal or concentric) in $88 \%$ cases, cardiomegaly in $92-100 \%$ cases, left ventricular hypertrophy $83-100 \%$ cases with or without left ventricular outflow tract obstruction and EKG anomalies $(13,14)$. The cardiomyopathy is related to intracellular glycogen accumulation leading to cellular hypertrophy and decreased systolic function (25).

Levine J.C. et al., in 2008, reported regression of left ventricular hypertrophy and improves the ejection fraction after enzyme replacement therapy with alglucosidase alfa (25).

Glycogen storage involves also the cardiac conduction system (A-V node and the His-bundle cells). The most common EKG anomalies are preexcitation syndromes (short PR in 10\% cases, delta waves) on 24 hours ECG Holter (26), Wolff-Parkinson-White syndrome $(27,28)$, atrio-ventricular blocks and bundle branch block in $4 \%$ cases $(26,29)$.

The pathogenic mechanism involved in Wolf Parkinson White syndrome is not fully understood. Several hypotheses have been put forward to explain the anomalie: the conduction system is directly affecting by the intracellular accumulation of glycogen or anulus fibrosus is anatomically interrupted by accumulation of glycogen. $(28,17)$ Some authors reported elevated plasma BNP (B-type natriuretic peptide) which seems to be related to the severity of cardiac disease (30).

The non classical form of PD with infantile onset has less severe clinical manifestations which becomes evident after 1 year age.

Echocardiography reveale a stationary hypertrophic cardiomyopathy, less severe than in classic form of PD and the missing of obstruction or ventricular diastolic dysfunction (31).

Late-onset PD it can occur at any age by progressive muscular hypotonie and respiratory insufficiency. Some patients could have arteriopathy with aneurysm of the basilar and internal carotid arteries or dilation of the ascending aorta because the accumulation of glycogen in vascular muscle (32-34). The patients could be asymptomatic or have unspecific neurologic symptoms (transient ischemic attacks and oculomotor nerve paralysis) (33).

Some studies which have been published quite recently reported for adult a short PR interval in $10 \%$ cases and a decreased left ventricular systolic function in $7 \%$ cases (26). Anaesthesia for patients 
with PD could precipitate arrhythmias, cause vasodilation and reduce myocardial contractility (35).

Hypothyroidism and subclinical hypothyroidism have a higher prevalence in patients with lateonset PD as compared to the general population, which could complicate pre-exesting cardiac disease (36).

In conclusion, routine cardiac evaluation in $\mathrm{PD}$ by echocardiography is an essential part of the PD

\section{REFERENCES}

1. Winchester B., Vellodi A., Young E. The molecular basis of lysosomal storage diseases and their treatment. Biochem. Soc. Trans. 2000; 28 (2): 150-4

2. Meikle P.J., Hopwood J.J., Clague A.E. et al. Prevalence of lysosomal storage disorders. JAMA. 1999; 281(3):249-54

3. Schultz M.L., Tecedor L., Chang M., Davidson B.L. Clarifying lysosomal storage diseases. Trends in neurosciences. 2011; 34(8):401-410

4. Filocamo M., Morrone A. Lysosomal storage disorders: Molecular basis and laboratory testing. Human Genomics. 2011; 5(3):156-169

5. Ole Kristian Greiner-Tollersrud, Thomas Berg, Lysosomal Storage Disorders, http://www.ncbi.nlm.nih.gov/books/NBK6177/, accessed October 10, 2016

6. Michael C. Kruer, Robert D. Steiner, Lysosomal Storage Disease, http://emedicine.medscape.com/article/1182830-overview\#showall, accessed October 10, 2016

7. Matsuishi T., Yoshino M., Terasawa K., Nonaka I. Childhood acid maltase deficiency: a clinical, biochemical, and morphologic study of three patients. Arch. Neurol. 1984; 41: 47-52, https://omim.org/ entry/232300 accessed October 12, 2016

8. Ausems M.G., Verbiest J., Hermans M.P., et al. Frequency of glycogen storage disease type II in The Netherlands: implications for diagnosis and genetic counseling. Eur J Hum Genet 1999 Sep; 7(6): 713-6.

9. Di lorio G., Cipullo F., Stromillo L., et al. Adult-onset Pompe disease. Acta Myologica. 2011; 30(3):200-202

10. Mechtler T.P., Stary S., Metz T.F., et al. Neonatal screening for Iysosomal storage disorders: feasibility and incidence from a nationwide study in Austria, Lancet. 2012 Jan; 379(9813):335-41

11. Hirschhorn, Rochelle, Arnold J.J. Reuser. Glycogen Storage Disease Type II: Acid Alpha-Glucosidase (Acid Maltase) Deficiency. In: Scriver C, Beaudet A, Sly W, Valle D, editors. The Metabolic and Molecular Bases of Inherited Disease. 8th Edition. New York: McGraw-Hill; 2001; 3389-3420.

12. Jones H.N., Muller C.W., Lin M., et al. Oropharyngeal dysphagia in infants and children with infantile Pompe disease. Dysphagia. 2010 Dec; 25(4):277-83

13. van den Hout H.M., Hop W., van Diggelen O.P., et al. The natural course of infantile Pompe's disease: 20 original cases compared with 133 cases from the literature. Pediatrics. 2003; 112:332-40

14. Kishnani P.S., Hwu W.L., Mandel H., et al. A retrospective, multinational, multicenter study on the natural history of infantile-onset Pompe disease. J Pediatr. 2006a; 148:671-6

15. Leslie N., Tinkle B.T. Glycogen Storage Disease Type II (Pompe Disease) 2007 Aug 31 (Updated 2013 May 9). In: Pagon RA, Adam MP, Ardinger HH, et al., editors. GeneReviews ${ }^{\circledR}$ (Internet). Seattle (WA): University of Washington, Seattle; 1993-2016. Available from: https://www.ncbi.nlm.nih.gov/books/NBK1261/

16. Beek N.A., Hagemans M.L., Ploeg A.T., et al. Pompe disease (glycogen storage disease type II): clinical features and enzyme replacement therapy. Acta Neurol Belg. 2006; 106:82-86 management, can follow the disease progression and response to intervention. The echocardiography should be performed by a pediatric cardiologist experienced in PD. A 24 hour ambulatory ECG should be perform as a diagnosis baseline of PD to highlight possible arrhythmias.

Conflict of interest: none declared Financial support: none declared
17. Müller-Felber W., Horvath R., Gempel K., et al. Late onset Pompe disease: clinical and neurophysiological spectrum of 38 patients including long-term follow-up in 18 patients. Neuromuscul Disord. 2007; 17:698-706

18. Kishnani P.S., Steiner R.D., Bali D., et al. Pompe disease diagnosis and management guideline. Genet Med. 2006b; 8:267-88

19. Young S.P., Piraud M., Goldstein J.L., et al. Assessing disease severity in Pompe disease: the roles of a urinary glucose tetrasaccharide biomarker and imaging techniques. Am J Med Genet C Semin Med Genet. 2012 Feb 15; 160C(1):50-8

20. Winchester B., Bali D., Bodamer O.A., et al. Pompe Disease Diagnostic Working Group. Methods for a prompt and reliable laboratory diagnosis of Pompe disease: report from an international consensus meeting. Mol Genet Metab. 2008 Mar; 93(3):275-81.

21. Prater S.N., Banugaria S.G., Dearmey S.M., et al. The emerging phenotype of long-term survivors with infantile Pompe disease. Genet Med. 2012;14:800-10

22. Han S.O., Pope R., Li S., Kishnani P.S., et al. A beta-blocker, propranolol, decreases the efficacy from enzyme replacement therapy in Pompe disease. Mol Genet Metab. 2016 Feb; 117(2):114-9

23. Elliott P., Andersson B., Arbustini E., et al. Classification of the cardiomyopathies: a position statement from the European Society Of Cardiology Working Group on Myocardial and Pericardial Diseases. Eur Heart J. 2008 Jan; 29(2):270-6

24. van der Beek N.A., Soliman O.I., van Capelle C.I., et al. Cardiac evaluation in children and adults with Pompe disease sharing the common c.-32-13T>G genotype rarely reveals abnormalities. J Neurol Sci. 2008 Dec 15; 275(1-2):46-50

25. Levine J.C., Kishnani P.S., Chen Y.T., et al. Cardiac remodeling after enzyme replacement therapy with acid alpha-glucosidase for infants with Pompe disease. Pediatr Cardiol. 2008 Nov; 29(6):1033-42

26. Forsha D., Li J.S., Smith P.B., et al. Cardiovascular Abnormalities in Late Onset Pompe Disease and Response to Enzyme Replacement Therapy. Genetics in medicine: official journal of the American College of Medical Genetics. 2011; 13(7):625-631

27. Tabarki B., Mahdhaoui A., Yacoub M., et al. Familial hypertrophic cardiomyopathy associated with Wolff-Parkinson-White syndrome revealing type II glycogenosis, Arch Pediatr. 2002 Jul; 9(7):697-700

28. Arad M., Moskowitz I.P., Patel V.V., et al. Transgenic mice overexpressing mutant PRKAG2 define the cause of Wolff-ParkinsonWhite syndrome in glycogen storage cardiomyopathy. Circulation 2003; 107(22):2850-6

29. Kishnani P.S., Steiner R.D., Bali D., et al. Pompe disease diagnosis and management guideline. Genet Med. 2006 May; 8(5):267-88. Erratum in: Genet Med. 2006 Jun;8(6):382.

30. Soker M., Kervancioglu M. Plasma concent rations of NT-pro-BNP and cardiac troponin-I in relation to doxorubicin-induced cardiomyopathy and cardiac function in childhood malignancy. Saudi medical journal, 2005, vol. 26, no. 8, pp. 1197-1202

31. Slonim A.E., Bulone L., Ritz S., et al. Identification of two subtypes of infantile acid maltase deficiency. J Pediatr. 2000; 137:283-5 
32. El-Gharbawy A.H., Bhat G., Murillo J.E., et al. Expanding the clinical spectrum of late-onset Pompe disease: dilated arteriopathy involving the thoracic aorta, a novel vascular phenotype uncovered. Mol Genet Metab. 2011; 103:362-6

33. Sacconi S., Bocquet J.D., Chanalet S., et al. Abnormalities of cerebral arteris are frequent in patients with late-onset Pompe disease. J Neurol. 2010; 257:1730-3

34. Kretzschmar H.A., Wagner H., Hübner G., et al. Aneurysms and vacuolar degeneration of cerebral arteries in late-onset acid maltase deficiency. J Neurol Sci 1990; 98:169-83
35. Wang L.Y.J., Ross A.K., Li J.S., et al. Cardiac arrhythmias following anaesthesia induction in infantile -onset Pompe disease: a case series. Pediar Anesth 2007; 17:738 -748

36. Joseph Schneider, Lynn A. Burmeister, Kyle Rudser, Chester B. Whitley Jeanine Jarnes Utz, Hypothyroidism in late-onset Pompe disease, Molecular Genetics and Metabolism Reports, September 2016; (8), 24-27 